\title{
Uncarboxylated Osteocalcin Levels in Patients With Metabolic Syndrome and Their Association With Metabolic Parameters
}

\author{
Selda Celik ${ }^{\mathrm{a}}$, Aytekin Oguz ${ }^{\mathrm{a}}$, Mehmet Uzunlulua, b, Ibrahim Yilmaz, \\ Damla Kilic ${ }^{a}$, Gulsum Koroglu
}

\begin{abstract}
Background: Uncarboxylated osteocalcin (uOCN) synthesized by osteoblasts was shown to increase insulin secretion and peripheral insulin sensitivity in pancreatic islets. The present study evaluated the uOCN levels and its association with metabolic parameters in non-diabetic patients with metabolic syndrome (MetS) to test our hypothesis that $\mathrm{uOCN}$ levels may be lower in MetS, which is characterized by insulin resistance.
\end{abstract}

Methods: The study included 30 patients with MetS aged 18 years and above (13 male, 17 female, mean age $39.00 \pm 5.09$ years) and 30 healthy controls (15 male, 15 female, mean age $36.23 \pm 6.71$ years). Diabetics and post-menopausal women were excluded. The International Diabetes Federation criteria were used to define MetS. Groups were compared depending on their uOCN levels, and association of uOCN with metabolic parameters was assessed.

Results: Serum u0CN levels were $5.56 \pm 3.36 \mathrm{ng} / \mathrm{mL}$ in patients with MetS whereas they were $6.26 \pm 2.65 \mathrm{ng} / \mathrm{mL}$ in healthy controls $(\mathrm{P}=0.183)$. Serum uOCN levels showed a negative correlation with HbA1c and body mass index $(r=-0.308, P=0.017 ; r=-0.278, P$ $=0.032$, respectively) in all patients, and with waist circumference

Manuscript accepted for publication April 13, 2012

${ }^{a}$ Medeniyet University Goztepe Training and Research Hospital, Internal Medicine, Istanbul, Turkey

${ }^{\mathrm{b}}$ Corresponding author: Mehmet Uzunlulu, Rasimpasa Mahallesi, Meltem Sokak, Tamer Apartman, No: 10, Daire: 5, Kadikoy, Istanbul, Turkey. Email: mehmetuzunlulu@yahoo.com

doi: $10.4021 / \mathrm{jem} 87 \mathrm{w}$ $(\mathrm{r}=-0.190, \mathrm{P}=0.032 ; \mathrm{r}=-0.379, \mathrm{P}: 0.047$, respectively) in males.

Conclusions: Although the difference in uOCN levels between patients with MetS and control group was not statistically significant, a negative correlation of $\mathrm{uOCN}$ with $\mathrm{HbA} 1 \mathrm{c}$, body mass index and waist circumference may provide support to our hypothesis that uOCN levels may be lower in MetS.

Keywords: Insulin resistance; Metabolic syndrome; Uncarboxylated osteocalcin

\section{Introduction}

Metabolic syndrome (MetS) is a clustering of cardiovascular risk factors that consists of abdominal obesity, hypertension, atherogenic dyslipidemia, hyperglycemia, and prothrombotic and proinflammatory conditions [1]. Insulin resistance has been suggested as an underlying pathogenic factor for MetS [2]. It has been suggested that there is an association between insulin resistance and bone metabolism, in which osteocalcin $(\mathrm{OCN})$ released from osteoblasts in the bone may be involved [3]. It is reported that uncarboxylated form of osteocalcin (uOCN) acts on pancreatic beta cells to improve insulin production and secretion, leading to release of adiponectin from adipocytes, and enhancing glucose use by increasing insulin sensitivity of the muscle tissue $[4,5]$. In insulin resistant states such Type 2 diabetes and obesity, OCN levels were shown to be lower [6]. While it has been reported that serum total OCN levels were lower in patients with MetS [7], no report exists about the uOCN levels. The present study compared serum uOCN levels in non-diabetic patients with MetS with healthy volunteers to test the hypothesis that uOCN levels may also be lower in MetS, which is characterized by insulin resistance, and evaluated the association of serum uOCN levels with metabolic parameters.

\section{Materials and Methods}

The study included patients aged 18 years and above, who 
were monitored at the Internal Medicine Polyclinic of the Goztepe Training and Research Hospital, and who admitted to participate in the study. Informed consent from all participants, and approval from the local ethics committee (Decision No $1 /$ A dated 06/04/2010) were obtained. The study was conducted in compliance with the Helsinki Declaration.

\section{Inclusion criteria}

In the MetS group, patients who received a diagnosis of MetS in accordance with the International Diabetes Federation criteria (IDF); and in the control group, healthy individuals who presented to the hospital for physical check-up, and found to be healthy as a result of workups.

\section{Exclusion criteria}

Postmenopausal women, patients with Type 1 or Type 2 diabetes, those with thyroid (hypo-hyperthyroidism) or paratyhroid (hypo-hyperparathyroidism) dysfunctions, chronic hepatic, renal or heart failure, those who receive treatments affecting the insulin resistance (metformin, glitazon, steroid, etc.), those who used coumadin or any drugs affecting the metabolism of vitamin D or calcium at least for 3 months during a period of lifetime, and those with intestinal malabsorption, vitamin D insufficiency, osteoporosis or malignity.

Criteria proposed by IDF were used for the diagnosis of MetS: waist circumference $>94 \mathrm{~cm}$ (male) or $>80 \mathrm{~cm}$ (female) and presence of at least two of the following: blood pressure $\geq 130 / 85 \mathrm{mmHg}$ (or use of antihypertensive medication); fasting plasma glucose $\geq 100 \mathrm{mg} / \mathrm{dL}$ (or use of antidiabetic medication); fasting triglyceride $\geq 150 \mathrm{mg} / \mathrm{dL}$ or medical treatment for high levels of triglyceride; HDL cholesterol $<40 \mathrm{mg} / \mathrm{dL}$ (male) or $<50 \mathrm{mg} / \mathrm{dL}$ (female), or medical treatment for low HDL cholesterol levels [8].

\section{Study design}

Information on demographics, comorbid diseases, smoking and alcohol habits and drugs used were obtained for all individuals who met inclusion criteria and gave consent, and their anthropometric measurements and biochemical data were recorded. Groups were compared on their demographic, anthropometric, and biochemical data and serum uOCN levels. The MetS group was subdivided into two groups; normoglycemic ( $<100 \mathrm{mg} / \mathrm{dL})$ and dysglycemic (100 - $125 \mathrm{mg} /$ $\mathrm{dL}$ ), and they were compared on their serum uOCN levels. A correlation analysis was conducted to define the association between $\mathrm{uOCN}$ and metabolic parameters.

\section{Anthropometric measurements}

Blood pressure was measured on both arms of the patient in a seated position after at least 10 minutes of a relaxation period, using an appropriate sphygomanometer based on the Korotkoff Phase I and Phase V sounds. A second measurement was performed on the arm with higher blood pressure. A mean value was calculated from these two systolic and diastolic blood pressure determinations with at least 3 minutes of interval. The waist circumference was measured at the narrowest point of waist in the midway between spina iliaca anterior superior and lower rib margin in light expirium when the patient was standing. The body mass index (BMI) was calculated by dividing the body weight in kilograms by height in square meters $\left(\mathrm{kg} / \mathrm{m}^{2}\right)$.

\section{Biochemical measurements}

The results of measurements for fasting plasma glucose, urea, creatinine, aspartate aminotransferase, alanine transaminase, LDL cholesterol, HDL cholesterol, total cholesterol, triglycerides, uric acid, alkaline phosphatase, calcium, phosphor, albumin, thyroid stimulating hormone, free T4, parathormone, $25(\mathrm{OH})$ vitamin $\mathrm{D} 3$ and $\mathrm{HbA} 1 \mathrm{c}$ were recorded from the patient files. Blood samples were taken from patients after a fasting period of 12 hours to determine the serum uOCN levels between 8.30 and 10.00 am. These samples were centrifuged at $4000 \mathrm{rpm}$ for 15 minutes, followed by separation of serums, and storage at -20 degrees. A solution of $100 \mathrm{mg} / \mathrm{mL}$ barium sulfate $\left(\mathrm{BaSO}_{4}\right)$ was prepared after serums were appropriately thawed. The $100 \mu \mathrm{l}$ $\mathrm{BaSO}_{4}$ solution and $100 \mu \mathrm{l}$ serum sample were mixed for 30 minutes at $4{ }^{\circ} \mathrm{C}$, followed by centrifugation at $10000 \mathrm{~g}$ for 2 minutes at $4{ }^{\circ} \mathrm{C}$. The uOCN was assessed from the resulting supernatant, which was obtained depending on difference in affinities to $\mathrm{BaSO}_{4}$, by electrochemiluminescence immunoassay (Elecsys 1010®, Roche, Weinheim, Germany) using the N-MID osteocalcin kit [9].

\section{Statistical analysis}

NCSS (Number Cruncher Statistical System) 2007 and PASS 2008 Statistical Software (Utah, USA) was used for statistical analyses. In addition to descriptive statistical methods (mean, standard deviation), Oneway Anova test was used for comparison of quantitative data between groups in comparing parameters with normal distribution, and Tukey's HDS test was used for determination of the group that was significantly different than others. Intergroup comparisons of parameters without normal distribution were made using the Kruskal Wallis test and determination of the group that was significantly different than the other was made using the Mann Whitney U test. Intergroup comparisons of parameters with normal distribution were made with the Student's t test. Qui-square test and Fisher's exact qui-square test were used for comparison of qualitative data. Spearman's rho correlation analysis was used for analysis of associations between parameters. Results were evaluated at a significance level of 


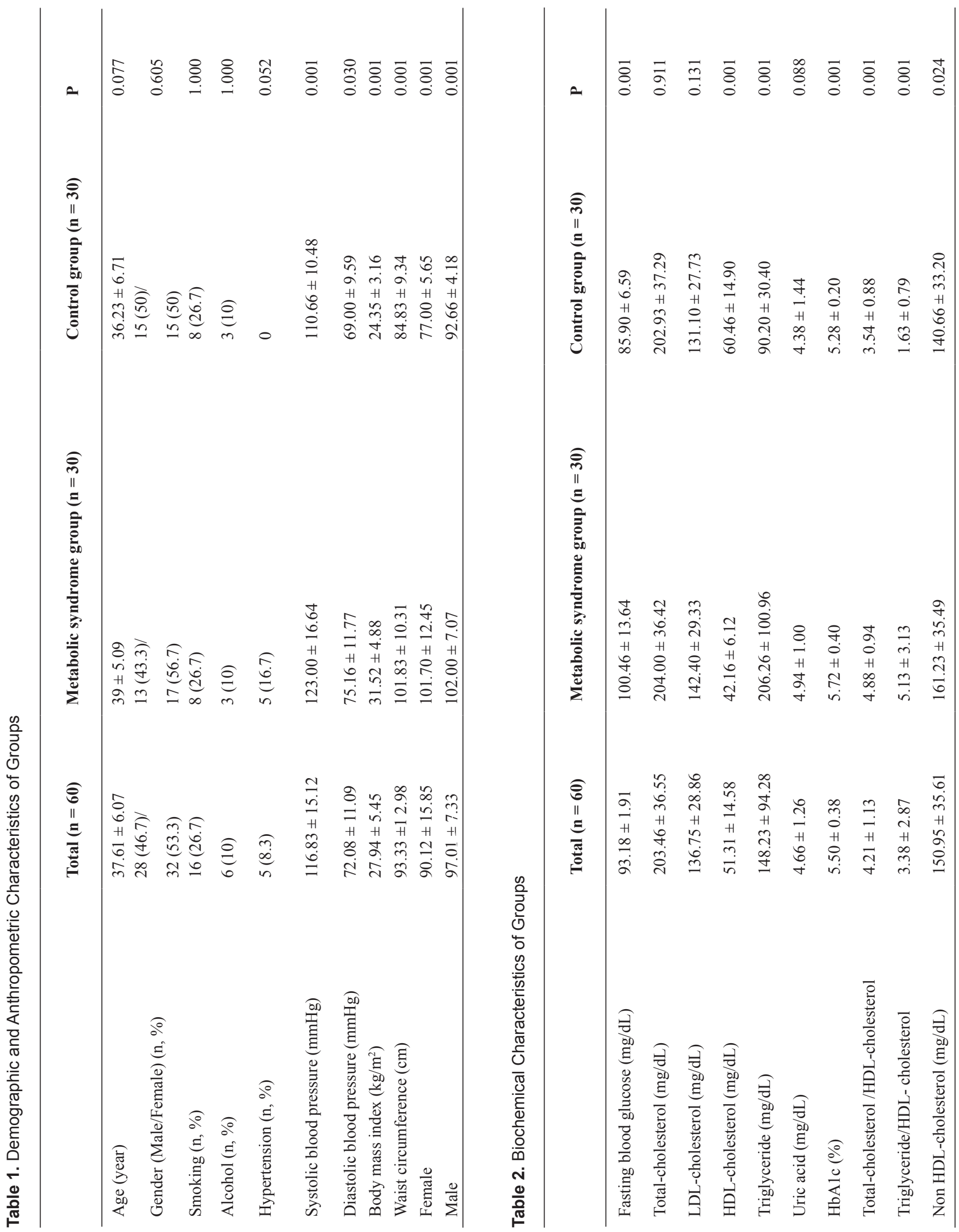




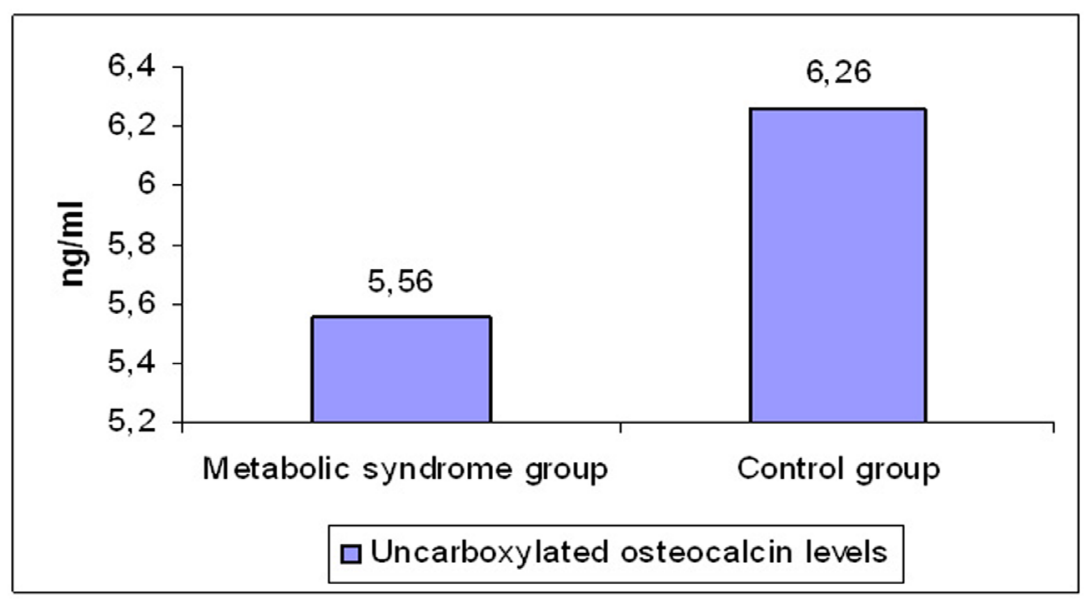

Figure 1. Comparison of serum uOCN levels between the groups.

$\mathrm{P}<0.05$

\section{Results}

A total of 60 individuals (28 male, 32 female, mean age $37.61 \pm 6.07$ years) were included in the study. The MetS group consisted of 30 patients (13 male, 17 female, mean age $39 \pm 5.09$ years), while there were 30 individuals (15 male, 15 female, mean age $36.23 \pm 6.71$ years) in the control group.

Demographic and anthropometric characteristics of participants are shown in Table 1.

Characteristics of age and sex, and frequency of smoking and alcohol intake were similar between groups ( $\mathrm{P}>$ 0.05 for all). The mean systolic and diastolic blood pressure, $\mathrm{BMI}$ and waist circumference were higher in MetS group compared to the control group ( $\mathrm{P}<0.05$ for all).
Biochemical characteristics of groups are shown in Table 2 .

Fasting blood glucose $(\mathrm{P}<0.01)$, triglycerides $(\mathrm{P}<$ $0.01), \mathrm{HbA1c}(\mathrm{P}<0.01)$, total cholesterol/HDL cholesterol $(\mathrm{P}<0.01)$, triglycerides/HDL cholesterol $(\mathrm{P}<0.01)$ and nonHDL cholesterol $(\mathrm{P}<0.05)$ levels were higher in the MetS group, and HDL cholesterol levels were lower compared to the control group $(\mathrm{P}<0.01)$. There was no significant difference in uric acid levels between the groups $(\mathrm{P}>0.05)$.

Comparison of serum uOCN levels between the groups is shown in Figure 1. The uOCN levels showed no significant difference between MetS and control groups (5.56 \pm 3.36 vs. $6.26 \pm 2.65, \mathrm{P}=0.183)$. Comparison of $\mathrm{uOCN}$ levels between patients with normoglycemic or dysglycemic MetS and control group is provided in Figure 2, uOCN levels showed no significant difference between the patients with normoglycemic, and dysglycemic MetS and the control group $(5.51 \pm 3.32,5.61 \pm 3.53,6.26 \pm 2.65$, respectively, $\mathrm{P}$

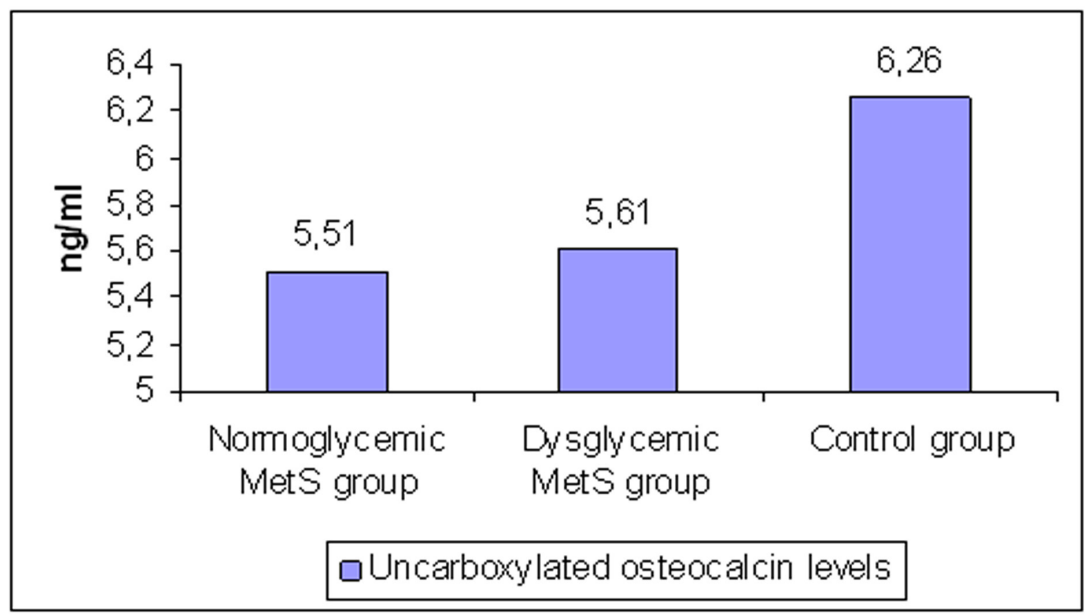

Figure 2. Comparison of uncarboxylated osteocalcin levels between patients with normoglycemic or dysglycemic metabolic syndrome and control group. MetS: metabolic syndrome. 
$=0.413)$.

Serum uOCN levels showed a negative correlation with HbAlc and BMI $(r=-0.308, P=0.017 ; r=-0.278, P=0.032$, respectively) in all patients, and with waist circumference $(\mathrm{r}=-0.190, \mathrm{P}: 0.032 ; \mathrm{r}=-0.379, \mathrm{P}=0.047$, respectively $)$ in males.

\section{Discussion}

Based on the results of the present study, it appears that the negative correlation of $\mathrm{uOCN}$ with $\mathrm{HbA} 1 \mathrm{c}$, body mass index and waist circumference support our hypothesis that uOCN levels may be lower in MetS although no statistically significant difference was found in uOCN levels between the patients with MetS and the control group.

While it has been already reported that serum total OCN levels were lower in patients with MetS [7], which is characterized by insulin resistance, no report has been published so far about the uOCN levels. The present study tested the hypothesis that levels of uOCN, the uncarboxylated form of serum OCN, may be lower in MetS, and compared the serum $\mathrm{uOCN}$ levels in non-diabetic patients with MetS with healthy volunteers, evaluating the association between serum $\mathrm{uOCN}$ levels and metabolic parameters. As a result, the fact that uOCN levels were lower (statistically not significant) in patients with MetS, both normoglycemic and dysglycemic, compared to the control group, and that uOCN showed a negative correlation with $\mathrm{HbA} 1 \mathrm{c}$, body mass index and waist circumference can be interpreted in favor of our hypothesis that uOCN levels may be lower in MetS.

A study by Shea et al [10] reported a negative correlation between carboxylated OCN and total OCN levels and homeostasis model assessment of insulin resistance (HOMA-IR) despite lack of any correlation between OCN and HOMAIR. This result further reinforces the significance and interpretation of our findings, that is to say, general opinion on a potential association between OCN and insulin resistance is directly related with lack of any assessment of $\mathrm{uOCN}$ in the studies performed. However, OCN exerts its effect on insulin production and insulin sensitivity through uOCN. Therefore, assessment of $\mathrm{uOCN}$ is more significant in determination of insulin resistance than assessment of total OCN and carboxylated OCN.

OCN synthesized by osteoblasts undergo vitamin Kdependent carboxylation, and binds to hydroxyapatite, being stored in the bone matrix. The uncarboxylated form acts like a hormon, with an action on pancreatic beta cell, adipose tissue and muscle, increasing the insulin sensitivity. The portion not stored is released into the circulation. Carboxylation of the molecule increases its affinity to hydroxyapatite. Due to this chemical feature, it was possible to differentiate the uncarboxylated form from the total OCN in circulation. The uOCN measurement was described by Price et al with indi- rect methods [11]. Although many studies used uOCN measurements from the supernatant by immunoassay technique following the incubation with hydroxyapatite, this method raises some concerns as it may non-specifically bind to hydroxyapatite. $\mathrm{BaSO}_{4}$ is an alternative to this procedure. The $\mathrm{BaSO}_{4}$ technique utilizes differences of binding of carboxylated and uOCN forms in affinity to $\mathrm{BaSO}_{4}$ [9]. An uOCN kit was also developed to allow measurement of uOCN levels, which also utilizes differences in affinity to hydroxyapatite. Therefore, concerns about the indirect method also maintain their presence in the use of a direct kit. The present study represents the first in the literature, which measured the uOCN levels in patients with MetS through $\mathrm{BaSO} 4$ precipitation.

In a study by Yeap et al [7], OCN level was found to be lower in male patients with MetS, and OCN level was shown to be negatively related with waist circumference, level of glucose, level of triglycerides and HOMA-IR. However, in a study by Saleem et al [12] with female patients with MetS, comparing the OCN levels, OCN levels showed a negative corelation with BMI, fasting blood glucose, fasting insulin, triglycerides, HOMA-IR and leptin, but a positive correlation with adiponectin. In the present study, uOCN levels showed a negative correlation with $\mathrm{HbA} 1 \mathrm{c}$ and $\mathrm{BMI}$ in all patients, and with waist circumference in male patients.

It has been reported that $\mathrm{OCN}$ levels increase with age, and they are higher in women of all age groups, particularly during the postmenopausal period [13]. The negative correlation found between advanced age and uOCN in male and female patients with MetS in the present study provides support to this finding. In a study with men and premenopausal and postmenopausal women with Type 2 diabetes [14], OCN levels were higher in postmenopausal women than in men and premenopausal women. Exclusion of postmenopausal women in the present study may have resulted in a more accurate assessment of uOCN levels.

The weak aspects of our study included the lack of HOMA-IR measurements as insulin levels were not assessed and the small study group. The strong aspects of our study included exclusion of postmenopausal cases, mean age less than 40 years, exclusion of patients with Type 2 diabetes, and use of $\mathrm{BaSO}_{4}$ method.

In conclusion, uOCN levels of patients with MetS showed a statistically not significant reduction compared to the control group in relation with insulin resistance. There was a negative correlation between uOCN levels and $\mathrm{HbA1c}$ and BMI in all patients, and waist circumference in male patients. This finding supports the relationship between uOCN and insulin resistance. Further large studies are needed to elucidate the relationship between MetS and uOCN levels.

\section{References}

1. Grundy SM, Brewer HB, Jr., Cleeman JI, Smith SC, Jr., 
Lenfant C. Definition of metabolic syndrome: Report of the National Heart, Lung, and Blood Institute/American Heart Association conference on scientific issues related to definition. Circulation. 2004;109(3):433-438.

2. Reaven G. The metabolic syndrome or the insulin resistance syndrome? Different names, different concepts, and different goals. Endocrinol Metab Clin North Am. 2004;33(2):283-303.

3. Fukumoto S, Martin TJ. Bone as an endocrine organ. Trends Endocrinol Metab. 2009;20(5):230-236.

4. Ferron M, Hinoi E, Karsenty G, Ducy P. Osteocalcin differentially regulates beta cell and adipocyte gene expression and affects the development of metabolic diseases in wild-type mice. Proc Natl Acad Sci U S A. 2008;105(13):5266-5270.

5. Lee NK, Sowa H, Hinoi E, Ferron M, Ahn JD, Confavreux C, Dacquin R, et al. Endocrine regulation of energy metabolism by the skeleton. Cell. 2007;130(3):456469.

6. Kindblom JM, Ohlsson C, Ljunggren O, Karlsson MK, Tivesten A, Smith U, Mellstrom D. Plasma osteocalcin is inversely related to fat mass and plasma glucose in elderly Swedish men. J Bone Miner Res. 2009;24(5):785791.

7. Yeap BB, Chubb SA, Flicker L, McCaul KA, Ebeling PR, Beilby JP, Norman PE. Reduced serum total osteocalcin is associated with metabolic syndrome in older men via waist circumference, hyperglycemia, and tri- glyceride levels. Eur J Endocrinol. 2010;163(2):265-272.

8. Alberti KG, Zimmet P, Shaw J. Metabolic syndrome-a new world-wide definition. A Consensus Statement from the International Diabetes Federation. Diabet Med. 2006;23(5):469-480.

9. Sokoll LJ, O’Brien ME, Camilo ME, Sadowski JA. Undercarboxylated osteocalcin and development of a method to determine vitamin K status. Clin Chem. 1995;41(8 Pt 1):1121-1128.

10. Shea MK, Gundberg CM, Meigs JB, Dallal GE, Saltzman E, Yoshida M, Jacques PF, et al. Gamma-carboxylation of osteocalcin and insulin resistance in older men and women. Am J Clin Nutr. 2009;90(5):1230-1235.

11. Price PA, Williamson MK, Lothringer JW. Origin of the vitamin K-dependent bone protein found in plasma and its clearance by kidney and bone. J Biol Chem. 1981;256(24):12760-12766.

12. Saleem U, Mosley TH, Jr., Kullo IJ. Serum osteocalcin is associated with measures of insulin resistance, adipokine levels, and the presence of metabolic syndrome. Arterioscler Thromb Vasc Biol. 2010;30(7):1474-1478.

13. Epstein S, Poser J, McClintock R, Johnston CC, Jr., Bryce G, Hui S. Differences in serum bone GLA protein with age and sex. Lancet. 1984;1(8372):307-310.

14. Zhou M, Ma X, Li H, Pan X, Tang J, Gao Y, Hou X, et al. Serum osteocalcin concentrations in relation to glucose and lipid metabolism in Chinese individuals. Eur J Endocrinol. 2009;161(5):723-729. 